\title{
Angio-Tumoral Laminin Expression in Human Gastrointestinal Adenocarcinomas
}

\author{
P. Tonino*, H.J. Finol*, C. Hidalgo** and L. Sosa*** \\ *Center for Electron Microscopy, Faculty of Sciences, Central University of Venezuela, Apdo. \\ 76963, El Marques 1070, Caracas, Venezuela \\ **Structural Biology Department, Venezuelan Institute for Scientific Research, Caracas, Venezuela \\ ***Department of Histology, Faculty of Medicine, Central University of Venezuela, Caracas
}

Angiogenesis and the extracellular matrix are fundamental to tumor progression from in situ invasive and metastatic disease [1]. In a previous work, we showed ultrastructural alterations of vascular basement membrane in different regions of human gastrointestinal tumors. Laminin, a major glycoprotein integrated into basement membranes, is observed in angiogenesis and tumorigenesis [2,3]. Although many immunohistochemical studies have been performed documenting the laminin distribution in invasive neoplasms [3], scarce data are available on laminin expression in the microvasculature of gastrointestinal malignant tumors during angiogenesis. To date, the localization of laminin in the tumor microvasculature in different regions of human gastrointestinal adenocarcinomas remains poorly defined. The aim of the present study was to determine the immunolocalization of laminin in microvessels in the stroma of human gastrointestinal adenocarcinomas, to analyze qualitatively the role of this basement membrane component during tumor-associate angiogenesis.

Specimens from the center of the tumor, periphery and lymph node metastasis were obtained immediately after surgical removal, from thirteen patients with a histopathological diagnosis of gastrointestinal adenocarcinoma. For indirect immunoperoxidase staining, paraffin sections were incubated with anti-human laminin antibody (polyclonal, diluted 1:50 in PBS). At the ultrastructural level, a post-embedding immunogold technique was used to localize laminin in the blood vessels. Tissue samples were fixed with $4 \%$ paraformaldehyde and $0.02 \%$ glutaraldehyde in Millonig buffer $\mathrm{pH} 7.4$, dehydrated in a ethanol series and embedded in LR-White resin. Thin sections on nickel grids were incubated with the same antibody (diluted 1:25 in PBS with 3\% BSA and 0.1\% Tween 20), and after staining in uranyl acetate and lead citrate were observed in a Hitachi H-7100 EM.

Immunohistochemical staining demonstrated that laminin expression in the angio-tumoral basement membrane was uniformly distributed, discontinuous (Figs.1, 2) or absent (Fig. 1). A considerable heterogeneity of laminin expression within each region and tumor was also observed. Laminin expression were more frequently positive in the superficial layer of tumor lessions, but were infrequently positive in the deeper layer. Immunogold labeling frequently showed an irregular distribution of laminin expressed in the basement membrane of blood vessels (Fig. 3), and in the endothelial cytoplasm, while in some capillaries laminin immunoreactivity was absent.

These results suggest that laminin expression in the tumor vasculature could reflect a variety of structural alterations in angiogenesis. The irregular or discontinuous laminin expression could be associated to an incomplete differentiation of the tumor blood vessels. In the different regions of the gastrointestinal adenocarcinomas the prominent staining of laminin in the vascular basement membrane may facilitate tumor progression and invasion, as have been observed in other tumors 
during angiogenesis [4]. Laminin expression in the endothelial cytoplasm of blood vessels suggests that endotelial cells contribute to the synthesis of this protein deposited in the blood vessel wall.

\section{References}

[1] L.C. Lugassy et al., J. Cutan. Pathol. 26 (1999) 78.

[2] P. Tonino et al., V IEMC, Venezuela (1999).

[3] S. Paku and N. Paweletz. Lab. Invest. 65 (1991) 334.

[4] R.E. Hewit et al., Br. J. Cancer 75 (1997) 221.

[5] H.G. Hagerdon and A.G. Nerlich. Acta Otolaryngol. 120 (2000) 891.

[6] This research was supported by a grant from CDCH of UCV (03-26-362/99).

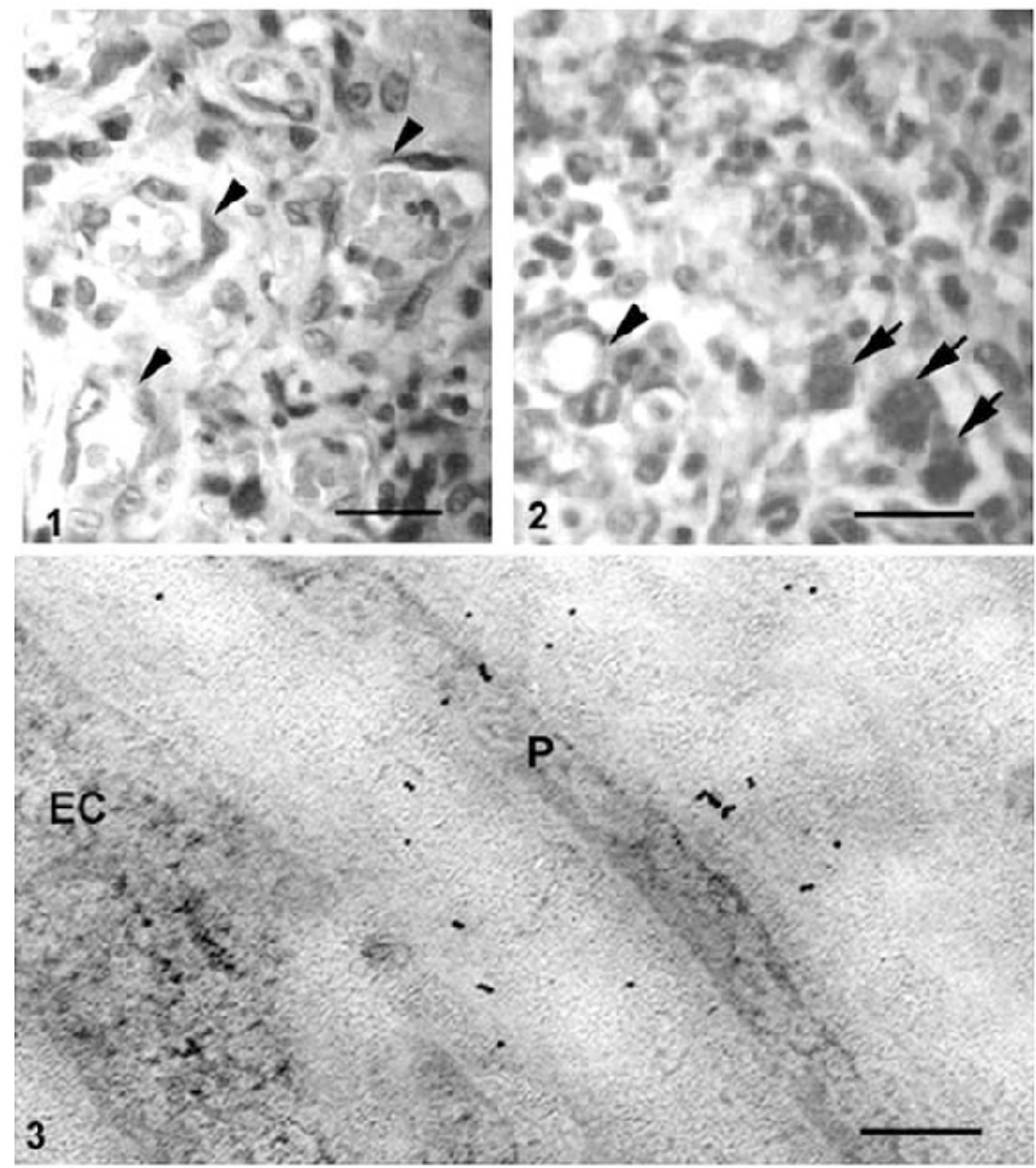

FIG. 1. Section of gastrointestinal adenocarcinoma stained with laminin antibody demonstrated in the basement membrane of blood vessels (arrowheads). Note the discontinuous distribution of laminin. Bar=12.5 $\mu \mathrm{m}$.

FIG. 2. In this section of gastrointestinal adenocarcinoma, continuous staining with laminin antibody is observed in a capillarie basement membrane (arrowhead), and a strong immunoreaction in some tumor cells (arrows) is also seen. Bar $=12.5 \mu \mathrm{m}$.

FIG. 3. Immunogold localization of laminin with an irregular distribution into the basement membrane of an endotelial cell (EC) and around the pericyte $(\mathrm{P})$. Bar $=0.25 \mu \mathrm{m}$. 